\title{
The Automated System to Detecting the Fabric Defect
}

\author{
Raswanth Rajhen.M ${ }^{1}$, Y.Suresh ${ }^{2}$, Karthick.K ${ }^{3}$ \\ PG Scholar, Information Technology, Sona College of Technology, Salem, India ${ }^{1}$ \\ Professor, Information Technology, Sona College of Technology, Salem, India ${ }^{2}$ \\ Assistant Professor, Information Technology, Sona College of Technology, Salem, India ${ }^{3}$
}

\begin{abstract}
The major role of this paper is inspection of fabrics. The fabric defect detection is carried out by manually in textile manufacturing industry. In manual work during which some of fabric defects are very small and undistinguishable. The important problems is inspection of fabric by human eye. Can be identified only monitoring by the skilled labour. The maximum number of the textile manufacturing industry in India performed the defect detection by many number of labour. To increase the fabric quality in industry to introduced the automated system. The automated fabric defect detection system detect defects definitely and identify them based on their physical appearance would naturally improve. To increase the customer satisfaction and reduce the costs associated with the quality. In recent periods many research are done on automated system to detect the fabric defect based on image processing techniques. These techniques takes much amount of time for processing the images to detect the fault. On this research, to overcome the above limitation, introduced the automated system based on deep learning algorithms to detect the defect. The proposed system efficiently detect the fabric defects with improved accuracy compared with other system.
\end{abstract}

Keywords: Fabric; Defect Detection; Automated; Manual; Textile

\section{INTRODUCTION}

Fabric defect detection is important technique used in the textile industry. The fabric defect detection important, because it only improve the quality of fabric before deliver to the consumers. The consumer detect the defect after the purchasing the fabric. In next purchase the consumer will order to another company. When the industry market value become very low. The quality of product in market becomes low, it consider as second quality or third quality product. Before in textile industry manual fabric defect detection technique is used the method has been both time consuming. It take more number of labours for defect detection and also consume more money. The accuracy of defect detection is very low. Economically it is failure in industry [2]. So that the industry become automate, the fabric Automated inspection systems are more needed in the textile manufacturing industry. The quality control of fabric in textile manufacturing industry is a major problem. Thus, automated defect detection systems show a great part in the quality of fabrics. To increase the efficiency to use the Open MP (Open Multi-Processing) is an Application Programming Interface (API)

\section{FABRIC DEFECTS}

The Fabric are used to manufacture many types and sets of products in the textile manufacturing industry. The defects can occur

Due to:

Scratch

Hole

Poor finishing

Machine faults

Colour bleeding

Defect image

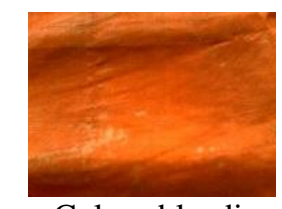

a. Colour bleeding 
Vol. 8, Issue 1, January 2019

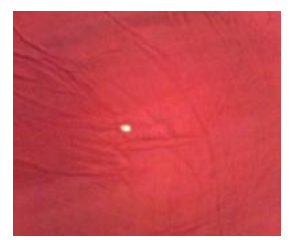

b. hole

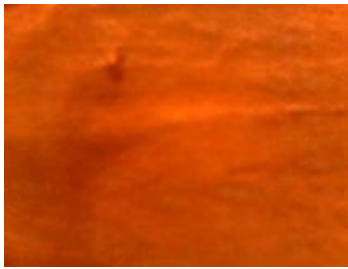

c. Machine faults

III. OPEN MP

Open MP is an implementation of multithreading, a method of parallelizing whereby a master thread(a series of instruction executed consecutively)fork a specified number of slave thread and the system divides a task among them. The threads then run parallel, with the runtime environment assigning threads to different processors. The section of code that is destined to run in similar is patent accordingly, with a compiler directive that will cause the threads to form before the section is executed. Each thread has an id attached to it which can be obtained using a function (called omp_get_thread_num()). The thread id is an integer, and the master thread has an id of 0. After the execution of the parallelized code, the threads join back into the master thread, which continues onward to the end of the program. By default, each thread executes the parallelized section of code independently. Work-sharing constructs can be used to divide a task among the threads so that each thread executes its allocated part of the code. Both task parallelism and data parallelism can be achieved using Open MP in this way. Can be achieved using Open MP in this way.

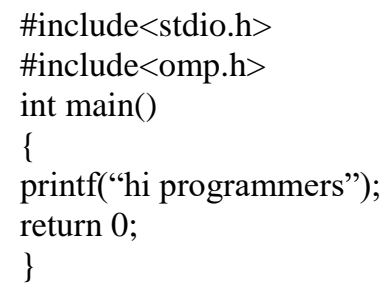

Use flag -fopenmp to compile using GCC:

\$ gcc-fopenmp hi.c-o hi

Output on a computer with two cores, and thus two threads:

hi programmers

hi programmers

\section{BRIEF LITERATURE SURVEY}

In general, fabric study is done on the base of digital images of the fabric. Instead, there are nearly works (Ciamberlini et al. [1]) based on the optical Fourier transform directly obtained from the fabric with optical devices and a laser beam. Digital image handling techniques have been progressively applied to textured examples are study over the last ten years. Some authors have considered defect detection on textile materials. Kang et al. [5, 6] studied textile samples from the pictures obtained from transmission and reflection of light to determine its knitting pattern. Tsai and Hu [3] used Fourier transforms of hard plane fabric picture as the set of inputs to an artificial neural network for fabric defect detection. The skilled neural network to classify four types of defects: missing pick, missing end, oil fabric stains and broken fabric. In a recent paper have also used wavelet packet bases and an artificial neural network for the stated goals. Wavelets had been before applied to fabric study by Jasper et al. [1, 5]. Justify the features in terms of their unfair qualities considering the problems which lie in the feature extraction process. We use statistical approach to extract the features. [6] 
Vol. 8, Issue 1, January 2019

V. METHODOLOGY

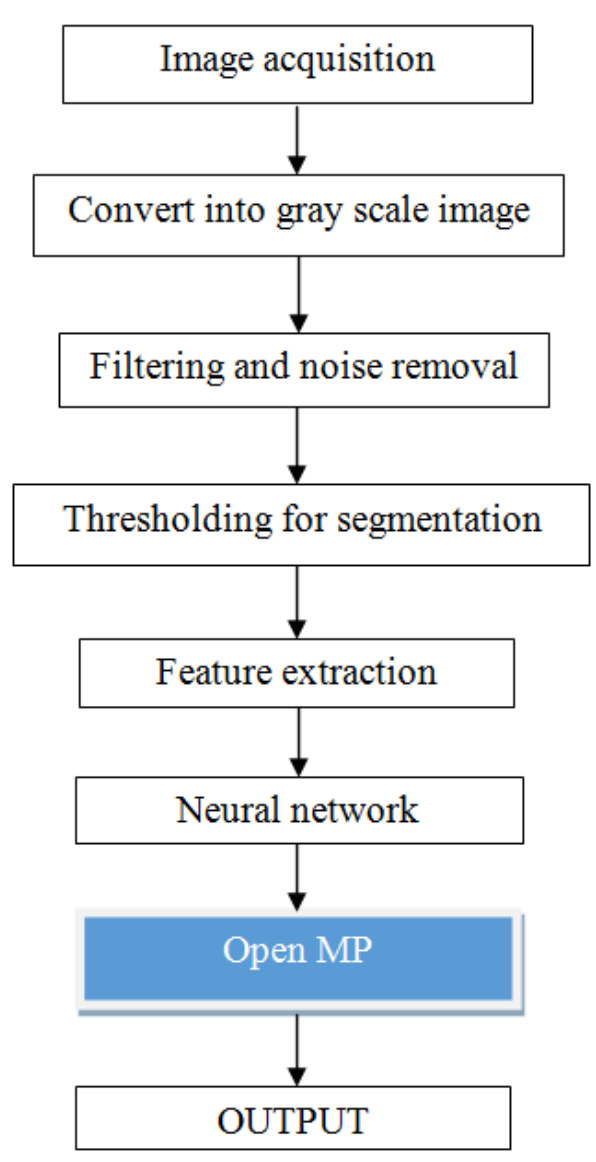

CONCLUSION

In this paper, we propose artificial neural network for patterned fabric inspection using open MP. Firstly images acquired, pre-processed and statistical features to be extracted. The extracted features are input to BPN classifier for further matching process. It can produce high accuracy and more efficient than total success rate of fabric identification s $87.5 \%$. Normal automatic inspection system

\section{REFERENCES}

[1]. Priya S, Kumar TA, Paul V. A novel approach to fabric defect detection using digital image processing, Signal Processing, Communication, Computing and Networking Technologies (ICSCCN), 2011 International Conference on IEEE, 2011, 228-232.

[2]. Chen T, Wu, HR. Space variant median filters for the restoration of the impulse noise corrupted images, IEEE Transaction Circuits syst.II, Analog Digital Signal Process 2001; 48(8):784-789.

[3]. Mak KL, Peng P, Yiu KFC. Fabric defect detection using morphological filters, International Journal of Emerging Technology and Advanced Engineering, Elsevier 2009; 27:1585-1592.

[4]. Chen T, Wu HR. Adaptive impulse detection using center -weigted median filters, IEEE signal process Letters 200115:1-3.

[5]. Anitha Ss, Radha V, Krishna PMHM, Enhanced Switching Median Filter For Denoising In 2D patterned Textile Image, International conference on modeling optimization and computing, Elsevier 2012; 38:3362-3372.

[6]. IOSR Journal of Electronics and Communication Engineering (IOSR- JECE) e-ISSN: 2278-2834,p- ISSN: 2278-8735.Volume 9, Issue 2, Ver. VIII (Mar - Apr. 2014), PP 70-76 